\title{
Pancreatic malignancy in the backdrop of chronic pancreatitis: How much to push the boundaries to achieve $\mathrm{RO}$ resection
}

Kunal Sadanand Joshi*1 ${ }^{* 1}$ Sisir Bodepudi ${ }^{1}$, Santhosh Kumar Ganapathi ${ }^{1}$, Chandrasekar Murugesan', Jagan Balu¹, Sankar Subramanian'

${ }^{1}$ Department of Surgical Gastroenterology, Sri Ramachandra Institute of

Higher Education and Research SRIHER (DU), Porur, Chennai, India

Received 3 July 2019; Accepted 1 March 2020

\begin{abstract}
Tumors of the body and tail of the pancreas are often more aggressive than tumors of the head and would have often undergone metastatic spread to other organs at the time of diagnosis. Most patients with carcinoma of the body and tail of the pancreas present at a late stage. Surgery is only indicated in those patients in whom there is no evidence of metastatic spread. Surgery is often not possible in cancers of the body and tail of the pancreas if the tumor invades celiac artery. Controversy exists regarding the margin status impact of microscopic resection margin involvement (R1) after pancreaticoduodenectomy (PD) for PDAC. There are reports indicating the rate of $\mathrm{R} 1$ resections increases significantly after PD if pathological examination is standardized. In this report, we present the case of a 56-year-old female who had undergone lateral pancreaticojejunostomy for chronic pancreatitis 8 years ago, but has now developed malignancy of the body and tail of the pancreas involving multiple organs. This patient underwent en bloc resection involving: 1. distal pancreatectomy with jejunal loop (lateral pancreaticojejunostomy) resection; 2. splenectomy; 3. left nephrectomy; 4. total gastrectomy; and 5. segmental colectomy with reconstruction by esophagojejunostomy, jejunojejunostomy, and colocolic anastomosis. The infrequent occurrence of tumor in the distal gland and advanced tumor stage at the time of diagnosis have both combined to produce therapeutic nihilism/dilemma in the minds of many surgeons. This report highlights the decision on how much to the push limits for multi-organ resection (en bloc resection with distal pancreatectomy, gastrectomy, splenectomy, colectomy, nephrectomy) with the intent of achieving RO status in spite of the complexity of surgery in selected patients.
\end{abstract}

Keywords: $R 1$ resection $\bullet$ pancreatic malignancy $\bullet$ chronic pancreatitis

\section{Introduction}

Malignancy arising in the background of chronic pancreatitis (CP) carries poor prognosis. It is generally a multifocal disease. The relationship between CP and pancreatic cancer has been the focus of multiple studies, many of which have indicated a strong association. The strength of this association varies between various causes of pancreatitis, with hereditary and tropical pancreatitis more likely to result in malignancy. The incidence of pancreatic cancer arising in $\mathrm{CP}$ accounts for only $0.1 \%-5 \%$ of all cases of PC, in tropical pancreatitis being $8.3 \%$ and in hereditary pancreatitis being $40 \%-$
$55 \%$. Tumors of the body and tail of the pancreas are often more aggressive than tumors of the head and would have often undergone metastatic spread to other organs at the time of diagnosis. Most patients with carcinoma of the body and tail of the pancreas present at a late stage and have an operability rate of $5 \%-10 \%$. Surgery is only indicated in those patients in whom there is no evidence of metastatic spread. Surgery is often not possible in cancers of the body and tail of the pancreas if the tumor invades coeliac artery. This report highlights the decision on how much to push the limits for multi-organ resection (en bloc resection with distal pancreatectomy, gastrectomy, splenectomy, colectomy, 
nephrectomy) with the intent of achieving R0 status in spite of the complexity of surgery in selected patients.

\section{Case report}

A 56-year-old female presented to the surgical gastroenterology outpatient department in Sri Ramachandra Medical College and Hospital with the chief complaints of pain in the left upper quadrant of abdomen since 2 months radiating to the back and occasional vomiting on and off.

Patient had history of lateral pancreaticojejunostomy done for CP for pancreatic stones 8 years back. Patient was a known diabetic and also had history of loss of weight and appetite.There was no history of hematemesis, melena or fever or jaundice. Patient presented to the hospital with a computerized tomography (CT) scan report obtained from a private center 2 months ago, which showed the tumor arising from the body and tail of the pancreas infiltrating the adjacent organs. On general physical examination, the patient was thin built and emaciated, weighing $35.3 \mathrm{~kg}$. On systemic per abdomen examination, there was a vague firm mass palpable in the left upper quadrant. Tenderness was present in the left upper quadrant. Previous surgical midline scar was seen and bowel sounds were normal.

Investigation revealed normal hematological parameters and liver and kidney function tests. CA 19-9 was $10.82 \mathrm{U} / \mathrm{ml}$. Upper gastrointestinal endoscopy revealed bulge in the proximal body of stomach with mucosal infiltration, and no evidence of varices. On contrast-enhanced computerized tomography (CECT), a mass was found arising from the distal pancreas, infiltrating the stomach, splenic hilum, left kidney encasing left renal vessels, splenic flexure of colon, and the jejunal loop (LPJ loop). The entire pancreas appeared atrophic and was found to be completely replaced by fat (Figure 1). ${ }^{18} \mathrm{~F}$-labeled fluoro-2-deoxyglucose Positron Emission Tomography (18F-FDG PET) scan revealed metabolically active lesion in the distal body and tail of the pancreas infiltrating the adjacent organs, with no evidence of metastasis.

This patient underwent diagnostic laparoscopy to rule out peritoneal disease, followed by laparotomy and en bloc resection of the tumor with: 1. distal pancreatectomy with jejunal loop (LPJ) resection; 2. splenectomy; 3. left nephrectomy; 4. total gastrectomy; and 5. segmental colectomy with reconstruction by esophagojejunostomy, jejunojejunostomy, and colocolic anastomosis. The intraoperative findings showed large growth arising from the body and tail of the pancreas infiltrating the left kidney hilum, left renal vessels, stomach (proximal body and

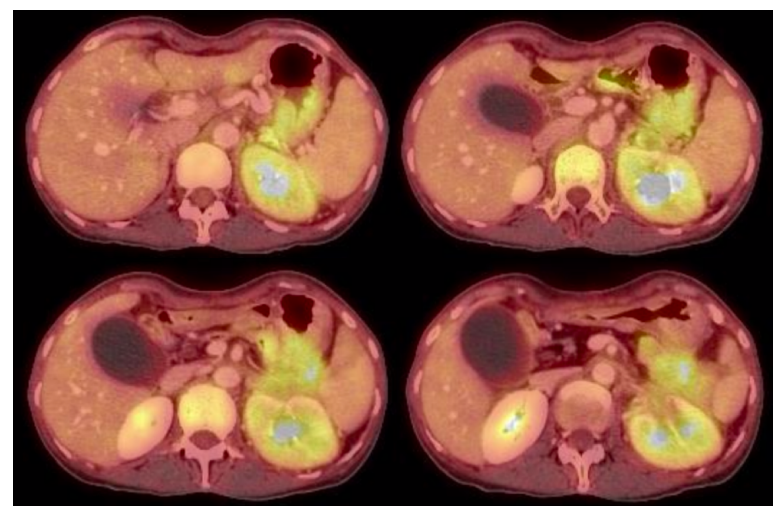

Figure 1:

posterior wall up to the lesser curvature), spleen, and splenic flexure of colon. The Roux loop of previous lateral pancreaticojejunostomy was noted. Liver was normal and no peritoneal deposits were noted (Figure 2).

Postoperative period was crucial. Orals were started on the seventh postoperative day. Nutrition was a major challenge. The endocrine and exocrine insufficiency was managed with enzyme supplements and elemental diet. Patient had delayed recovery and brittle diabetes.

Histopathology revealed pT3 pN0 G2 moderately differentiated adenocarcinoma with focal mucinous differentiation $(20 \%)$ of pancreas infiltrating the left kidney hilum, left renal vessels, stomach (proximal body and posterior wall up to the lesser curvature), spleen, and the splenic flexure of colon. Margins were free of tumor (Figures 3 and 4).

Patient was on follow-up up to 6 months after surgery, during which period she was on nutritional supplements and required management of brittle diabetes. Further follow-up is required to assess long-term recovery.

\section{Discussion}

Literatures have documented the clinical presence of extended resection of multiorgan pancreatic corpus and tail, portal vein resection, resection of the coeliac artery and left adrenal gland, subtotal gastrectomy, splenectomy, gastrojejunostomy (Roux-Y), oversewing of the pancreatic head, and duodenojejunostomy $[1,3,5,6,20]$. In this study, we discuss how much to push the boundaries/limits to achieve $\mathrm{R} 0$ resection by presenting a case study. Pancreatic cancer arising in case of CP accounts only for $0.1 \%-5 \%$ of all PC [21]. Local vascular invasion of the coeliac axis (CA) is often associated with pancreatic body and tail ductal adenocarcinomas. The surgical options include distal 

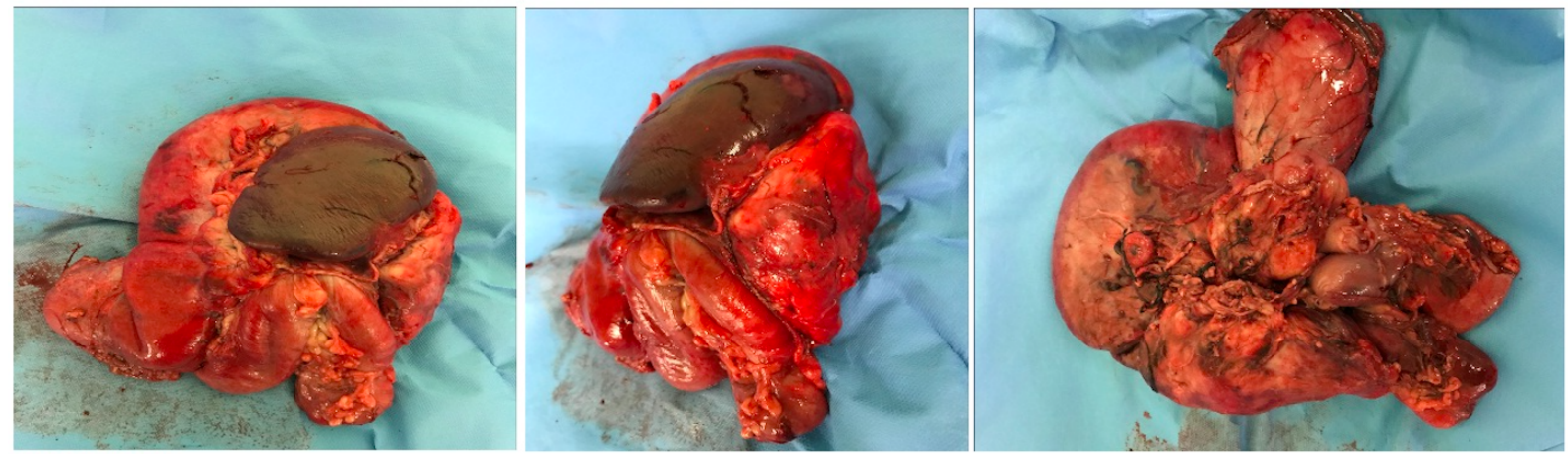

Figure 2:
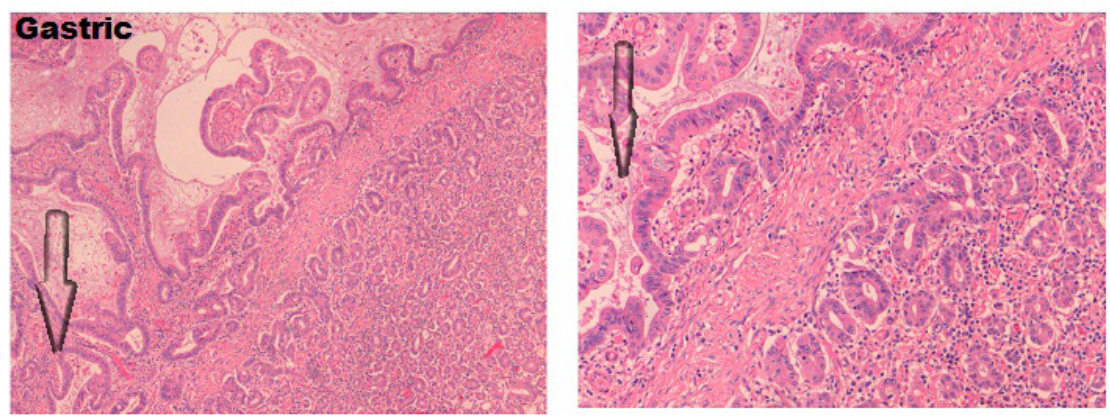

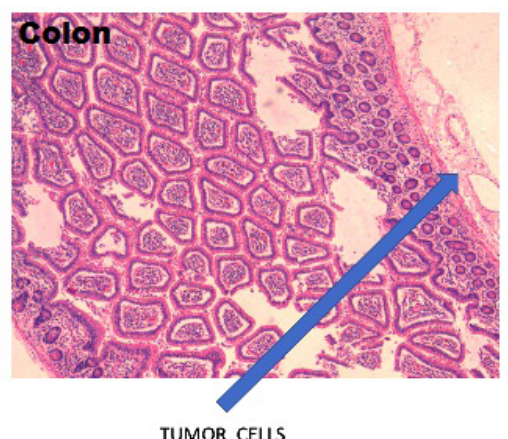

Low power

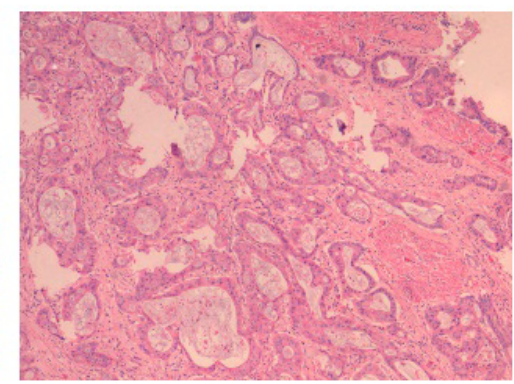

High power

Figure 3:

pancreatectomy and/or en bloc splenectomy including CA resection which relies on the presence of collateral arterial circulation through a gastroduodenal artery and intact pancreaticoduodenal arcade to preserve prograde hepatic arterial perfusion as described in Appleby procedure. Many of the studies focused on CP and its relation to pancreatic cancer have indicated a strong association, which varies depending upon the causes of pancreatitis, and stated that hereditary and tropical pancreatitis likely results in malignancy in a majority of cases. Whereas the incidence of pancreatic cancer is limited to only about $0.1 \%-5 \%$ of overall CP cases, the chance of cancer is up to $8.3 \%$ and $40 \%-55 \%$ in tropical and hereditary pancreatitis, respectively $[8-10,17]$. Most patients with carcinoma of the body and tail of the pancreas present at a late stage [19-21]. At our center, although it is not a common practice to adopt such an aggressive approach/radical surgery, it was decided after a multidisciplinary team (MDT) meeting that the best chance of a cure (to achieve R0 resection) would be possible only if multiorgan resection is attempted and consideration that the Positron Emission Tomography (PET) scan had ruled out distant metastasis. 

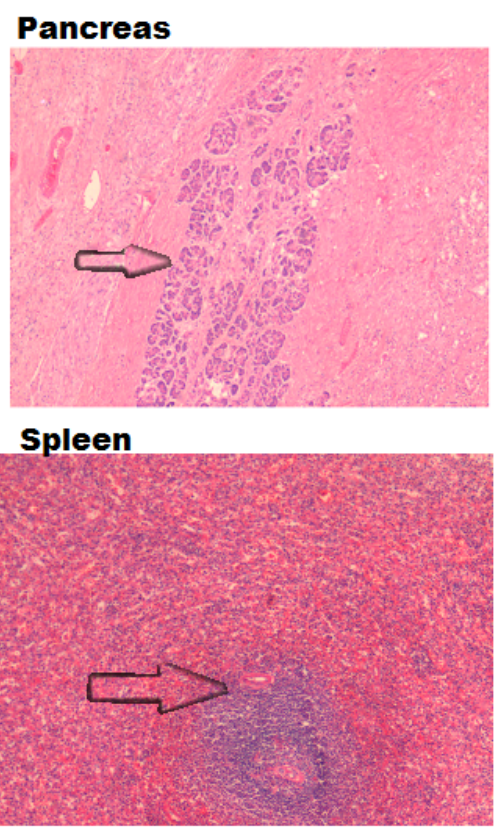
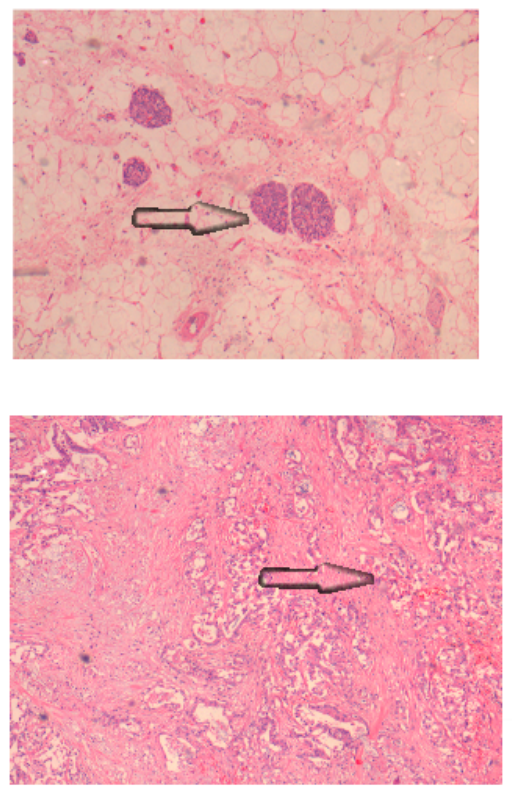

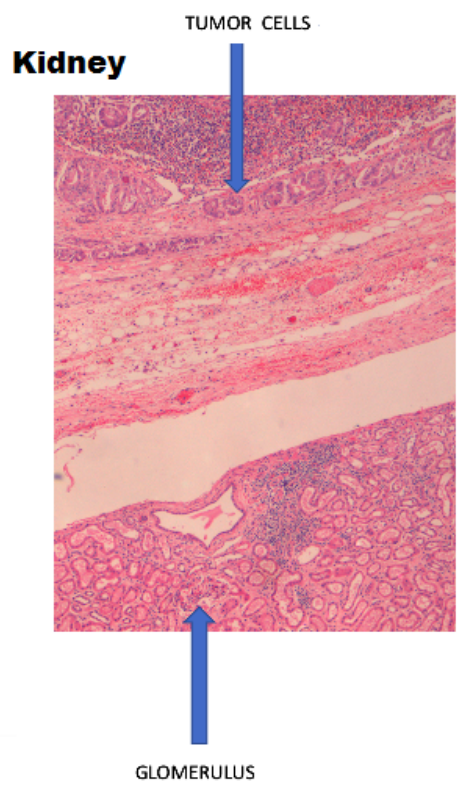

Figure 4:

\section{Conclusion}

The infrequent occurrence of tumor in the distal gland and the advanced tumor stage have both combined to produce therapeutic nihilism/dilemma in the minds of many surgeons at the time of diagnosis. Multiorgan resection (en bloc resection with distal pancreatectomy, gastrectomy, splenectomy) should be attempted with the intent of achieving RO status in spite of the complexity of surgery.

\section{References}

[1] Siegel R, Ma J, Zou Z, Jemal A. Cancer statistics, 2014. CA Cancer J Clin. 2014;64:9-29.

[2] Bilimoria KY, Bentrem DJ, Ko CY, Stewart AK, Winchester DP, Talamonti MS. National failure to operate on early stage pancreatic cancer. Ann Surg. 2007;246:173-80.

[3] Neoptolemos JP, Stocken DD, Dunn JA, Almond J, Beger HG, Pederzoli P, et al. Influence of resection margins on survival for patients with pancreatic cancer treated by adjuvant chemoradiation and/ or chemotherapy in the ESPAC-1 randomized controlled trial. Ann Surg. 2001;234:758-68.

[4] Menon KV, Gomez D, Smith AM, Anthoney A, Verbeke CS. Impact of margin status on survival

following pancreatoduodenectomy for cancer: the Leeds Pathology Protocol (LEEPP). HPB (Oxford). 2009;11:18-24.

[5] Hartwig W, Hackert T, Hinz U, Gluth A, Bergmann $\mathrm{F}$, Strobel O, et al. Pancreatic cancer surgery in the new millennium: better prediction of outcome. Ann Surg. 2011;254:311-9.

[6] Gnerlich JL, Luka SR, Deshpande AD, Dubray BJ, Weir JS, Carpenter DH, et al. Microscopic margins and patterns of treatment failure in resected pancreatic adenocarcinoma. Arch Surg. 2012;147:753-60.

[7] Raut CP, Tseng JF, Sun CC, Wang H, WolffRA, Crane $\mathrm{CH}$, et al. Impact of resection status on pattern of 
failure and survival after pancreaticoduodenectomy for pancreatic adenocarcinoma. Ann Surg. 2007;246:52-60.

[8] Butturini G, Stocken DD, Wente MN, Jeekel $H$, Klinkenbijl JH, Bakkevold KE, et al. Influence of resection margins and treatment on survival in patients with pancreatic cancer: metaanalysis of randomized controlled trials. Arch Surg. 2008;143: 75-83.

[9] Verbeke CS, Menon KV. Redefining resection margin status in pancreatic cancer. HPB (Oxford). 2009;11:282-9.

[10] Schlitter AM, Esposito I. Definition of microscopic tumor clearance $(\mathrm{r} 0)$ in pancreatic cancer resections. Cancers (Basel). 2010;2:2001-2010.

[11] Wagner M, Redaelli C, Lietz M, Seiler CA, Friess $H$, Buchler MW. Curative resection is the single most important factor determining outcome in patients with pancreatic adenocarcinoma. $\mathrm{Br} \mathrm{J}$ Surg. 2004;91:586-594.

[12] Kuhlmann K, de Castro S, van Heek T, Busch $\mathrm{O}$, van Gulik T, Obertop $\mathrm{H}$, et al. Microscopically incomplete resection offers acceptable palliation in pancreatic cancer. Surgery. 2006;139: 188-96.

[13] Westgaard A, Tafjord S, Farstad IN, Cvancarova $\mathrm{M}$, Eide $\mathrm{TJ}$, Mathisen $\mathrm{O}$, et al. Resectable adenocarcinomas in the pancreatic head: the retroperitoneal resection margin is an independent prognostic factor. BMC Cancer. 2008;8:5.

[14] Verbeke CS, Leitch D, Menon KV, McMahon MJ, Guillou PJ, Anthoney A. Redefining the R1 resection in pancreatic cancer. $\mathrm{Br} J$ Surg. 2006;93:12321237.

[15] Esposito I, Kleeff J, Bergmann F, Reiser C, Herpel $\mathrm{E}$, Friess $\mathrm{H}$, et al. Most pancreatic cancer resections are R1 resections. Ann Surg Oncol. 2008;15:16511660.

[16] Campbell F, Smith RA, Whelan P, Sutton R, Raraty M, Neoptolemos JP, et al. Classification of R1 resections for pancreatic cancer: the prognostic relevance of tumour involvement within $1 \mathrm{~mm}$ of a resection margin. Histopathology. 2009;55: 277-83.

[17] Jamieson NB, Chan NI, Foulis AK, Dickson EJ, McKay CJ, Carter CR. The prognostic influence of resection margin clearance following pancreaticoduodenectomy for pancreatic ductal adenocarcinoma. J Gastrointest Surg. 2013;17:511 521.
[18] Sobin LH, Gospodarowicz MK, Wittekind C. International Union Against Cancer. TNM classification of malignant tumours. 7th ed. Oxford: Wiley-Blackwell; 2009.

[19] College of American Pathologists. Protocol for the examination of specimens from patients with carcinoma of the exocrine pancreas [Internet]. Northfield, IL: College of American Pathologists [cited 2015 Jun 1]. Available from: http://www. cap. org/web/home/resources/cancer-reporting-tools/ cancer-protocol-templates.

[20] Latona, J.A., Lamb, K.M., Pucci, M.J. et al. J Gastrointest Surg (2016) 20: 300. https://doi. org/10.1007/s11605-015-3001-2

[21] Dhar P, Kalghatgi S, Saraf V. Pancreatic Cancer in Chronic Pancreatitis. Indian Journal of Surgical Oncology. 2015;6(1):57-62. doi:10.1007/s13193014-0373-9.

[22] Appleby Operation for Locally Advanced Tumour of the Pancreatic Body and Tail, Zentralbl Chir 2015; 140(2): 151-154 DOI: 10.1055/s-0035-1545837 